\title{
COMPLETE MOMENT CONVERGENCE FOR $(\alpha, \beta)$-MIXING RANDOM VARIABLES AND ITS APPLICATION
}

\author{
JIANGFENG HAO AND KAN CHEN
}

\begin{abstract}
In this paper, the complete moment convergence for weighted sums of $(\alpha, \beta)$-mixing random variables is investigated. The result improves and extends the corresponding one of $\mathrm{Wu}$ et al. (2017). As a corollary, the complete convergence for weighted sums of $(\alpha, \beta)$-mixing random variables is obtained, which is applied to establish the complete consistency for the P-C estimator in a nonparametric regression model.
\end{abstract}

Mathematics subject classification (2010): 60F15, 62G20.

Keywords and phrases: $(\alpha, \beta)$-mixing random variables, complete moment convergence, P-C estimator, nonparametric regression model, complete consistency.

\section{REFERENCES}

[1] Bai Z.D., Cheng P.E., Marcinkiewicz strong laws for linear statistics, Statistics \& Probability Letters, 46(2), 2000, 105-112.

[2] Baum L.E., Katz M., Convergence rates in the law of large numbers, Transactions of the American Mathematical Society, 120(1), 1965, 108-123.

[3] Benedetti J.K., On the nonparametric estimation of regression functions, Journal of the Royal Statistical Society: Series B (Statistical Methodology), 39, 1977, 248-253.

[4] BRADLEY R.C, BRYC W., Multilinear forms and measures of dependence between random variables, Journal of Multivariate Analysis, 16, 335-367.

[5] CAI Z.W., Strong consistency and rates for recursive nonparametric conditional probability density estimates under $(\alpha, \beta)$-mixing conditions, Stochastic Processes and Their Applications, 38, 1991, 323-333.

[6] Chen P.Y., Bai P., Sung S.H., The von Bahr-Esseen moment inequality for pairwise independent random variables and applications, Journal of Mathematical Analysis and Applications, 419(2), $1290-1302$.

[7] Chen P.Y., Sung S.H., On complete convergence and complete moment convergence for weighted sums of $\rho^{*}$-mixing random variables, Journal of Inequalities and Applications, Volume 2018, Article ID 121, 2018, 16 pages.

[8] CHOw Y.S., Delayed sums and Borel summability of independent, identically distributed random variables, Bulletin of the Institute of Mathematics, Academia Sinica, 1(2), 1973, 207-220.

[9] CHOW Y.S., On the rate of moment convergence of sample sums and extremes, Bulletin of the Institute of Mathematics, Academia Sinica, 16(3), 1988, 177-201.

[10] ERdös, P., On a theorem of Hsu and Robbins, The Annals of Mathematical Statistics, 20(2), 1949, 286-291.

[11] GaO P., Strong stability of $(\alpha, \beta)$-mixing sequences, Applied Mathematics-A Journal of Chinese Universities, 31(4), 2016, 405-412.

[12] Hsu P.L., Robbins H., Complete convergence and the law of large numbers, Proceedings of the National Academy of Sciences U.S.A., 33, 1947, 25-31.

[13] LiAng H.Y., Jing B.Y., Asymptotic properties for estimates of nonparametric regression models based on negatively associated sequences, Journal of Multivariate Analysis, 95, 2005, 227-245.

[14] Liang H.Y., Li D.L., RosalSKY A., Complete moment and integral convergence for sums of negatively associated random variables, Acta Mathematica Sinica, English Series, 26(3), 2010, 419-432. 
[15] LU C.R., Lin Z.Y., Limit Theory for Mixed Dependent Variables, Beijing, Science Press of China, 1997.

[16] Priestley M.B., Chao M.T., Non-parametric function fitting, Journal of the Royal Statistical Society: Series B (Statistical Methodology), 34, 1972, 385-392.

[17] SAMURA S.K., WANG X.J., WU Y., Consistency properties for the estimators of partially linear regression model under dependent errors, Journal of Statistical Computation and Simulation, 89(13), 2019, 2410-2433.

[18] Shao Q.M., Limit theorems for the partial sums of dependent and independent random variable, Hefei, University of Science and Technology of China, 1989, 1-309.

[19] SHEN A.T., Complete convergence for weighted sums of END random variables and its application to nonparametric regression models, Journal of Nonparametric Statistics, 28(4), 2016, 702-715.

[20] Shen A.T., Xue M.X., Volodin A., Complete moment convergence for arrays of rowwise NSD random variables, Stochastics: An International Journal of Probability and Stochastic Processes, 88 (4), 2016, 606-621.

[21] SHEN Y., ZHANG Y.J., Strong limit theorems for $(\alpha, \beta)$-mixing random variable sequences, Journal of University of Science and Technology of China, 41(9), 2011, 778-795.

[22] Sung S.H., Complete convergence for weighted sums of $\rho^{*}$-mixing random variables, Discrete Dynamics in Nature and Society, Volume 2010, Article ID 630608, 2010, 13 pages.

[23] Sung S.H., On the strong convergence for weighted sums of $\rho^{*}$-mixing random variables, Statistical Papers, 54, 2013, 773-781.

[24] Wang X.J., Hu S.H., Complete convergence and complete moment convergence for martingale difference sequence, Acta Mathematica Sinica, English Series, 30, 2014, 119-132.

[25] Wang X.J., Shen A.T., Chen Z.Y., Hu S.H., Complete convergence for weighted sums of NSD random variables and its application in the EV regression model, TEST, 24(1), 2015, 166-184.

[26] Wu Q. Y., Probability Limit Theory for Mixing Sequences, Beijing, Science Press of China.

[27] Wu Y., WANG X.J., Hu S.H., Complete moment convergence for weighted sums of weakly dependent random variables and its application in nonparametric regression model, Statistics \& Probability Letters, 127, 56-66.

[28] WU Y., WANG X.J., Equivalent conditions of complete moment and integral convergence for a class of dependent random variables, RACSAM, 112, 2018, 575-592.

[29] Wu Y., WANG X.J., BALAKRISHNAN N., On the consistency of the P-C estimator in a nonparametric regression model, Statistical Papers, 61, 2020, 899-915.

[30] Wu Y.F., CABrea M.O., Volodin A., Complete convergence and complete moment convergence for arrays of rowwise END random variables, Glasnik Matematički, 49(69), 2014, 449-468.

[31] YANG S.C., WANG Y.B., Strong consistency of regression function estimator for negatively associated samples, Acta Mathematicae Applicatae Sinica, 22(4), 1999, 522-530.

[32] YU C.Q., Convergence theorems of weighted sum for $(\alpha, \beta)$-mixing sequences, Journal of Hubei University (Natural Science), 38(6), 2016, 477-487. 